\title{
CONDITIONS, RÉSULTATS ET PERSPECTIVES D'UTILISATION DU COTON GÉNÉTIQUEMENT MODIFIÉ (COTON BT) DANS LES PAYS EN DÉVELOPPEMENT
}

\author{
Michel FOK *
}

\begin{abstract}
La commercialisation à grande échelle des variétés génétiquement modifiées, a exacerbé le débat sur leur pertinence pour les pays en développement (PED). Dès 1997-1998, l'utilisation effective de coton génétiquement modifié (CGM) dans des pays présentés comme des PED (Afrique du Sud, Chine, Inde principalement) apporte des éléments pour dépasser la dimension théorique du débat. Cette utilisation concerne uniquement le coton Bt.

La littérature scientifique est assez abondante pour indiquer les effets positifs de l'utilisation du CGM : efficacité technique, rentabilité économique et impacts positifs sur le plan environnemental et de la santé publique, avec la réduction des traitements chimiques sont à l'origine d'une certaine pression pour étendre l'utilisation du CGM dans les PED.

L'objet de cet article est de procéder à une analyse plus détaillée des expériences existantes pour : nuancer les succès rapportés et rappeler la dépendance des résultats avec les conditions de diffusion et de production; tempérer les craintes sur l'imposition inéluctable d'une situation de monopole des semenciers qui capteraient seul le bénéfice de l'utilisation du CGM; souligner le changement, induit par l'utilisation du CGM, de la structure des coûts de production dans les systèmes à faible intensification et qui accentue le risque financier de la production; indiquer l'impossibilité d'une rentabilité assurée pour tous les utilisateurs et donc la nécessité d'une
\end{abstract}

* CIRAD-UPR 10, TA 72/09. 
cohabitation avec du coton conventionnel; alerter sur la difficulté d'adapter la distribution des semences pour une telle cohabitation ; mettre en évidence le caractère excessif de l'optimisme et du pessimisme dans les anticipations des effets du CGM ; et souligner le caractère incomplet de ces anticipations qui ont négligé par exemple la modification du complexe parasitaire.

La commercialisation à grande échelle des variétés génétiquement modifiées, à partir de la campagne 1996-1997, a exacerbé le débat (PINSTRUP-ANDERSEN et SCHIOLER, 2001) sur la pertinence de ces variétés pour les pays en développement (PED). D'un côté, on les voyait comme la solution pour permettre aux PED de rattraper leur retard de productivité et de résoudre le problème de la faim dans le monde (Asian DEVElopment Bank, 2001, PraKash, 2004). De l'autre, les critiques ou suspicions étaient de plusieurs ordres. On relevait l'inadéquation des variétés disponibles pour contribuer à remédier au problème de la faim (MACDONALD, 2003). On soulignait les risques de soumettre les paysans pauvres au pouvoir de monopole des semenciers (MAZOYER, 2000) en leur faisant adopter une solution technique dont on mettait en doute l'efficacité. On alertait sur les risques environnementaux, liés à l'évolution de la faune ou de la flore en conséquence de l'utilisation des variétés génétiquement modifiées. Sur ce plan, on a plus particulièrement mis en avant la crainte de l'émergence de ravageurs résistants aux toxines Bt produites dans les plantes génétiquement modifiées (MYERS, 1999). Le débat est devenu tellement passionné, voire irrationnel parfois, que d'aucuns appellent à davantage de sérénité et de méthode pour cerner l'utilisation des OGM en agriculture (Conner, Glare et NAP, 2003 ; Kelemu, MahuKu, Fregene et al., 2003) en invitant à fonder les analyses sur les études de cas (GIANESSI, SiLVER, SANKULA et CARPENTER, 2002).

La commercialisation à grande échelle de cotonnier génétiquement modifié (CGM) dans les PED, ou considérés comme tels (l'Afrique du Sud et la Chine dès 1997-1998, et l'Inde en 2002), a concerné essentiellement les variétés résistantes à certains ravageurs incorporant un gène $\mathrm{Bt}$ (coton $\mathrm{Bt}$ ). Après dix ans d'utilisation, le recul permet d'apprécier les résultats obtenus.

Le CGM n'est cependant pas une nouveauté technologique habituelle. Son développement a nécessité des investissements privés très élevés. Sa diffusion est accompagnée de règles nouvelles et particulières pour son utilisation. Ce sont ces règles qui déterminent pour beaucoup les impacts, notamment économiques, de leur utilisation. C'est une certaine variation de ces règles entre les pays utilisateurs, méconnue ou insuffisamment prise en compte, qui peut expliquer la diversité des impacts obtenus. L'objet de cet article est d'évaluer ces impacts en relation avec les conditions de diffusion. Cette approche devrait permettre de mieux appréhender les perspectives d'utilisation du CGM dans les PED.

La deuxième partie est centrée sur l'analyse des innovations institutionnelles qui ont accompagné la diffusion du CGM dans le monde. La troisième partie 
propose une synthèse des résultats d'utilisation du CGM pour souligner les différences suivant les modes de production et les niveaux économiques des pays. La quatrième partie est une analyse rétrospective des effets anticipés, positifs ou négatifs, au regard des résultats aujourd'hui disponibles. La conclusion précise les conditions, en particulier économiques et organisationnelles, pour que l'utilisation soit réellement bénéfique aux PED.

\section{I - ANALYSE DES CONDITIONS D'UTILISATION DU CGM}

On recense aujourd'hui près de 90 millions d'hectares de cultures génétiquement modifiées. On recense environ 10 millions d'hectares de CGM, essentiellement dans sept pays (USA, Chine, Inde, Australie, Argentine, Mexique, Afrique du Sud). La diffusion a subi peu de retard dans les PED où c'est l'utilisation du CGM qui prédomine (tableau 1), l'utilisation de cultures vivrières génétiquement modifiées (maïs, soja, colza) y étant le plus souvent proscrite.

La diffusion de cultures génétiquement modifiées a été lancée avec la mise en œuvre de conditions ou de règles nouvelles qui imposent des engagements spécifiques aux utilisateurs concernés. Ces règles correspondent à de véritables innovations institutionnelles, c'est-à-dire des changements par rapport aux règles qui préexistaient dans le domaine de l'utilisation des semences. Lorsque ces innovations sont formalisées et transcrites dans des contrats très explicites, on peut parler d'innovation contractuelle. Il y a cependant une certaine diversité dans les conditions d'utilisation du CGM entre les pays concernés. Compte tenu de l'incidence de ces conditions sur les impacts d'utilisation du CGM, il est important de restituer la diversité et l'évolution en cours de ces conditions.

\section{1 - Une innovation contractuelle diversement appliquée}

Depuis son commencement, en 1996, la commercialisation du CGM s'est accompagnée d'une innovation institutionnelle, due à la multinationale Monsanto, appliquée d'abord aux États-Unis avant d'être étendue à d'autres pays.

L'innovation institutionnelle a consisté à obliger le paysan utilisateur à signer un contrat par lequel il accepte le paiement d'une redevance d'emploi et l'interdiction de l'utilisation de la production obtenue comme semences paysannes ${ }^{1}$. Dans le cas du coton $\mathrm{Bt}$, il doit aussi s'engager à installer une parcelle dite de refuge, semée avec des semences conventionnelles.

Par cette démarche contractuelle, le prix des semences n'est pas réellement différent de celui des semences de coton conventionnel mais la valeur de la redevance s'y ajoute. Ce système s'est cependant appliqué à des pays où les semences étaient déjà cédées au prix du marché, de sorte que la redevance représentait le seul surcoût. Ce n'est pas le cas dans tous les PED, comme les pays de l'Afrique Zone Franc, et cette différence est importante à considérer (HoFs, FOK, GOUSE et KiRSTEN, 2006).

1 - C'est-à-dire utilisation d'une partie de la production obtenue comme semences pour installer les cycles de culture suivants. 
Tableau 1 : Évolution des superficies de cultures d'ogm dans le monde

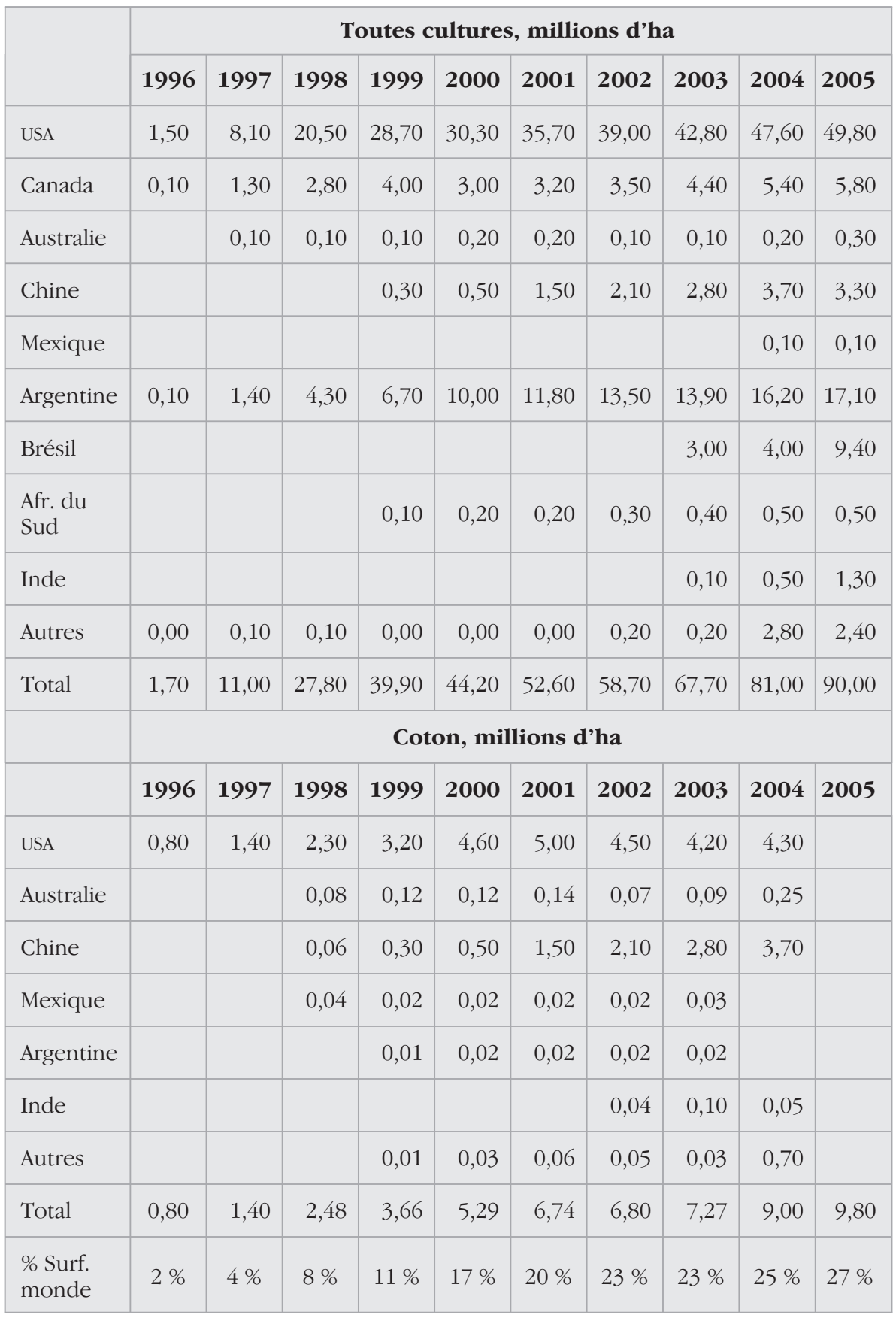

Sources : International Service for the Acquisition of Agri-biotech Applications (ISAA) et International Cotton Advisory Committee (ICAC). 
La redevance d'emploi est jugée généralement élevée ${ }^{2}$, même par les utilisateurs de pays industrialisés. Il n'est pas aisé de reconstituer les valeurs de la redevance d'emploi des semences de coton Bt dans les différents pays. Nous en proposons une première synthèse, qui reste imparfaite, en montrant aussi le différentiel du prix des semences dans les pays où la redevance n'est pas appliquée (tableau 2).

Tableau 2 : Variation du niveau de la redevance d'emploi et du différentiel de prix des semences de coton $\mathrm{Bt}$ (US\$/ha)

\begin{tabular}{|c|c|c|c|c|c|c|c|c|c|}
\hline & \multicolumn{6}{|c|}{ Redevance d'emploi } & \multicolumn{3}{|c|}{$\begin{array}{l}\text { Différentiel de prix } \\
\text { des semences } \\
\text { (Bt - non-Bt) }\end{array}$} \\
\hline & \multirow[b]{2}{*}{ USA } & \multirow[b]{2}{*}{$\begin{array}{c}\text { Aus- } \\
\text { tralia }\end{array}$} & \multicolumn{2}{|c|}{ South Africa } & \multirow[b]{2}{*}{$\begin{array}{l}\text { Mexi- } \\
\text { que }\end{array}$} & \multirow[b]{2}{*}{$\begin{array}{c}\text { Argen- } \\
\text { tine }\end{array}$} & \multirow{2}{*}{\begin{tabular}{|c|} 
Inde \\
$\begin{array}{c}\text { Hybri- } \\
\text { des }\end{array}$
\end{tabular}} & \multicolumn{2}{|c|}{ Chine } \\
\hline & & & $\begin{array}{l}\text { Dry, } \\
\text { small }\end{array}$ & $\begin{array}{l}\text { Irrigate, } \\
\text { large }\end{array}$ & & & & $\begin{array}{l}\text { Popu- } \\
\text { lation }\end{array}$ & $\begin{array}{c}\text { Hybri- } \\
\text { des }\end{array}$ \\
\hline 1996-1997 & 66 & 135 & & & & & & & \\
\hline $1997-1998$ & & & & & 61 & & & & \\
\hline 1998-1999 & & & & & 56 & & & & \\
\hline $1999-2000$ & & & & & & 75 & & 38 & \\
\hline 2000-2001 & & & & & & 85 & & 60 & \\
\hline $2002-2003$ & 80 & & 49 & 104 & & & 21 & & \\
\hline 2003-2004 & & 85 & & & & & & & \\
\hline $2004-2005$ & & & & & & & & 5 & 83 \\
\hline
\end{tabular}

Il y a une grande variation des niveaux de redevance exigée entre les pays où celle-ci s'applique. Cette variation est attribuée aux différences dans la réduction espérée d'utilisation des insecticides (CHAudhry, 2002). Plus élevée est l'espérance de réduction, plus coûteuse sera la redevance d'emploi de la technologie à payer. En d'autres termes, il est de bonne logique commerciale de vanter une forte économie en insecticides pour faire payer plus chère la technologie.

2 - Le paiement de la redevance d'emploi est généralement justifié par les investissements spécifiques dans le domaine des biotechnologies. On ne peut cependant comprendre le niveau élevé de la redevance sans connaître les sommes d'argent engagées dans les opérations associées de partenariat entre les grandes firmes impliquées ni les motivations financières de ces dernières. À titre indicatif, Monsanto et Delta \& Pineland ont signé, le 8 mai 1998, un accord permettant au premier d'absorber le deuxième. Cet accord a été invalidé par un jugement du 20 décembre 1999 pour non-conformité à la loi anti-trust des États-Unis. Cet échec conduisit Delta \& Pineland à réclamer compensation des dommages et intérêts à Monsanto, d'un montant d'un milliard de uS\$ (http://sec.edgar-online.com/2005). 
Le niveau de la redevance varie aussi dans le temps, dans un même pays. Aux États-Unis, la redevance a augmenté alors qu'en Australie les fermiers ont pu la faire baisser (cf. infra). Dans les pays où la redevance d'emploi ne s'applique pas, et mises à part les variétés hybrides, les différentiels de prix des semences sont d'un niveau plus faible que la redevance d'emploi. En Chine, le marché concurrentiel (FOK, LIANG, WANG et Wu, 2005) semble bien induire une baisse du différentiel des prix de semences, mais cette tendance semble s'inverser de nouveau avec l'offre de variétés hybrides pour capter une rente plus confortable.

La diffusion du CGM a associé dans la pratique Monsanto, firme de biotechnologie, et Delta \& Pineland Company, firme semencière disposant d'un certain portefeuille variétal. Il a découlé de ce partenariat, s'agissant du coton, une approche mondiale de la diffusion d'un nombre limité de variétés de CGM. La variété $N U C O T N 33 B$ par exemple a été commercialisée aux États-Unis, en Mexique, en Argentine, en Afrique du Sud et en Chine. Aux États-Unis et en Australie, les variétés respectivement appelées Bollgard et Ingard sont identiques. Cette démarche est discutable sur le plan agronomique car il est irréaliste d'attendre qu'un nombre limité de variétés puisse convenir à la diversité de conditions naturelles d'utilisation. La moindre performance, quantitative et/ou qualitative, de ces variétés a en effet été observée en Chine et au Mexique (XU, You, WANG et YANG, 2004 ; MAGAÑA, GARCIA, RODRIGUEZ et GARCIA, 1999).

On recense actuellement deux pays où la diffusion du CGM se fait sans que les paysans aient à payer une redevance d'emploi. L'exemple le plus significatif est la Chine où les paysans n'ont pas à s'engager formellement en signant un contrat (FOK, LIANG, WANG et Wu, 2005). Le coût de la technologie est alors intégré dans le prix des semences. Les paysans chinois ont également conservé la liberté d'utiliser les graines de leur production pour servir de semences, ce dont ils ne se privent pas. Dans les provinces utilisant essentiellement les variétés conventionnelles de CGM (" populations » non hybrides), près de $50 \%$ des paysans ne renouvellent pas leurs semences tous les ans (FOK, LIANG, WANG et Wu, 2005).

Le même cas de figure s'applique en Inde depuis la commercialisation, en 2002, mais avec la grande différence d'une diffusion exclusive de variétés hybrides F1 dont les semences doivent être renouvelées annuellement. Ce n'est pas forcément le cas (Russell et Deguine, 2006) et cela pourrait expliquer les grandes différences des résultats observés (BENNETT, IsMAËL et MORSE, 2005).

\section{2 - Une innovation institutionnelle en évolution}

La brouille quasi consommée entre les deux firmes alliées (LAws, 2004), Monsanto et Delta \& Pineland Company, semble accentuer une variation dans l'approche de Monsanto. Cette dernière est plus volontiers désireuse de donner licence à l'utilisation des gènes dans les variétés développées, par les pays d'utilisation. C'est le cas en Inde ${ }^{3}$, mais dans une démarche commerciale de diffusion exclusive de variétés hybrides.

3 - Mahyco Monsanto Biotech (India) Ltd est une entreprise à capitaux mixtes par laquelle Monsanto donne licence aux firmes semencières en Inde. En 2005, licence a été donnée à 19 firmes semencières à travers le pays. 
Le coût de la technologie est alors intégré directement dans le prix des semences. On constate aussi que le nombre de fournisseurs, essentiellement locaux, de variétés de CGM augmente (ANON, 2006a). La situation de monopole au profit d'une firme étrangère ne s'applique donc pas aux semences mais à la fourniture des gènes.

Les firmes biotechnologiques ajustent donc les innovations institutionnelles qui accompagnent l'utilisation du CGM, pour préserver leur rente biotechnologique. Cela est général dans la diffusion des oGm. Au Brésil, Monsanto a réussi à faire intervenir les négociants de soja nationaux en tant que collecteurs de royalties, à son profit.

\section{II - DES RÉSULTATS COMPLEXES MAIS PORTEURS D'ENSEIGNEMENTS}

Il n'est pas aisé de faire une synthèse des résultats rapportés sur l'utilisation du coton Bt dans le monde. Les résultats peuvent être obtenus en culture pluviale ou irriguée, dans des exploitations de tailles très variables, pour des années différentes, pour des régions différentes d'un même pays, pour des pays à niveaux de développement économique très distincts. Le cas de l'Afrique du Sud est typique, avec à la fois petite agriculture familiale (noire) et agriculture de grandes fermes (blanche), avec culture irriguée ou pas.

En dépit des réserves formulées ci-dessus, il est utile de réunir les résultats pour en faciliter la synthèse. Nous l'avons fait, de manière encore imparfaite et incomplète, en réunissant les résultats des pays suivant la taille des exploitations et le recours à l'irrigation, un pays comme l'Afrique du Sud pouvant se retrouver dans les deux groupes de pays (tableau 3 et tableau 4).

\section{1 - Les limites des méthodes d'analyse}

Les méthodes par lesquelles les résultats sont obtenus et rapportés sont aussi fréquemment discutables. Les résultats présentés par Matin QAIM (QUAIM, 2003) et ayant contribué à l'idée d'une très forte rentabilité du coton Bt en Inde, correspondent surtout à une extrapolation de résultats d'expérimentation fournis par la firme Monsanto. Lorsque les résultats sont obtenus effectivement en situation d'utilisation réelle chez les paysans, les comparaisons de rentabilité ont rarement lieu entre des parcelles de coton Bt et de coton conventionnel cultivées dans les mêmes fermes. Même quand cela est le cas, les variétés de coton Bt et de coton conventionnel ne se différencient pas seulement par ce seul caractère (on dit qu'elles ne sont pas isogéniques). Il est donc abusif d'attribuer les différences observées au seul caractère Bt, comme cela est couramment fait.

Il y a donc une difficulté technique pour élaborer une méthode irréprochable de comparaison, et cette difficulté est accentuée par la rapidité de diffusion du coton Bt dans certains pays (en Chine notamment) : on y trouve très peu ou pas de parcelles de référence en coton conventionnel. 
Par contre d'autres biais sont plus directement liés à l'approche méthodologique du chercheur. Le choix de la méthode de budget partiel conduit à supposer, souvent, que seuls les postes de dépense en semences et en protection phytosanitaire sont modifiés. Cette hypothèse s'avère erronée car on constate que les paysans tendent à intensifier davantage et à accorder plus de soin, lorsque l'espérance de rendement est augmentée par l'utilisation du coton Bt. Cela est observé dans plusieurs pays et notamment en Inde (ORPHAL, 2005).

La surestimation peut découler aussi de l'hypothèse d'un prix identique à la vente du coton Bt et du coton conventionnel. Cette hypothèse est discutable car la qualité de la fibre du coton Bt peut être moins bonne pour diverses raisons, comme au Mexique (Magaña, Garcia, Rodrigues, Garcia, 1999) ou en Inde (OrPHAL, 2005). Aux États-Unis, la moindre qualité du CGM a été mise en cause, obligeant Monsanto à se défendre (KERBY, 2001).

Une autre erreur d'estimation interpelle davantage car elle est plus systématique dans les pays où les parcelles refuges sont imposées (très peu protégées contre les ravageurs). Le rendement obtenu sur ces parcelles refuges est obligatoirement bien plus faible. Or ces parcelles sont rarement prises en compte dans la comparaison de rentabilité, ce qui surestime l'impact économique du gène Bt.

La grande variation de résultats entre les pays et à l'intérieur d'un même pays tend à alimenter surtout les polémiques. Pourtant, l'application de toute nouveauté technique ne peut pas conduire uniquement à des résultats uniformes. Pour cerner les perspectives d'extension de l'utilisation du coton Bt, l'analyse de l'hétérogénéité des résultats et des facteurs responsables est fondamentale. Par commodité, la discussion des résultats est organisée en fonction de deux groupes, avec les limites que cela comporte.

\section{2 - En grande production irriguée, une incidence négligeable sur le gain de productivité}

Dans le groupe de pays aux exploitations agricoles de grande taille, le recours à l'irrigation explique la grande variation dans les rendements obtenus (tableau 3). Cela est manifeste en Afrique du Sud mais aussi aux États-Unis où la production peut être irriguée ou pas. En Argentine, la production n'est pratiquement jamais irriguée, le niveau de rendement est bien plus faible qu'en Australie où l'irrigation est systématique.

Aux États-Unis, en Australie, mais aussi partiellement en Afrique du Sud, la production intensive en usage d'intrants conduit à des rendements élevés. C'est une production très capitalistique. Le temps consacré à la culture d'un hectare aux États-Unis peut être estimé à douze heures (à comparer aux 100-150 jours/ha dans les pays de l'Afrique Zone Franc). Cette indication permet de souligner que le gain de productivité (du travail) dans ces pays découle plus de l'augmentation du rendement et/ou de la réduction des coûts de production.

Dans ces pays, le gain de rendement est faible voire nul, et le nombre de traitements insecticides est seulement réduit de 2 à 3 (tableau 3). En Australie, il 


\begin{tabular}{|c|c|c|c|c|c|c|c|c|c|c|c|c|c|c|}
\hline \multirow{4}{*}{ 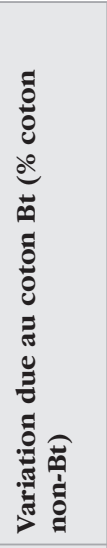 } & 㟧 & & & & & & & & & $\begin{array}{l}\text { वे } \\
\text { ते }\end{array}$ & $\begin{array}{l}20 \\
\infty \\
i n\end{array}$ & $\begin{array}{l}\alpha 0 \\
\infty \\
-\end{array}$ & $\begin{array}{l}d 0 \\
\vdots \\
-1\end{array}$ & \multirow{2}{*}{ 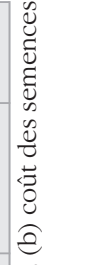 } \\
\hline & 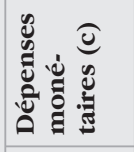 & & & & & & & & & & & & & \\
\hline & 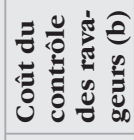 & $\begin{array}{l}0 \\
\infty \\
\cdots \\
i \\
0 \\
1\end{array}$ & & $\stackrel{\partial}{a}$ & $\begin{array}{l}\circ \\
\infty\end{array}$ & $\stackrel{2}{\stackrel{7}{7}}$ & वे & 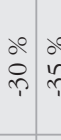 & $\hat{r}$ & & & $\begin{array}{l}\text { do } \\
\text { i }\end{array}$ & $\stackrel{\partial}{i}$ & 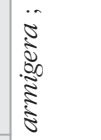 \\
\hline & 岇 & $\begin{array}{l}\partial 0 \\
\exists \\
\pi \\
n\end{array}$ & $\begin{array}{l}\propto 0 \\
\infty\end{array}$ & $\begin{array}{l}\text { do } \\
\text { ì }\end{array}$ & @ & $\begin{array}{l}0 \\
\infty \\
\infty\end{array}$ & $\stackrel{\partial}{-}$ & $\begin{array}{lll}0 & 0 \\
m & \text { in }\end{array}$ & $\overbrace{}^{\circ}$ & $\begin{array}{l}\text { o } \\
\text { nै }\end{array}$ & $\begin{array}{l}00 \\
\vec{n} \\
\vec{n}\end{array}$ & $\begin{array}{l}\circ \\
\stackrel{0}{\beth}\end{array}$ & 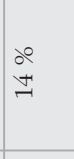 & 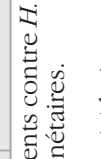 \\
\hline 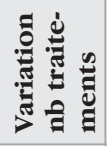 & & $\stackrel{-}{i}$ & & $\begin{array}{l}0 \\
\stackrel{n}{n}\end{array}$ & î & $\stackrel{\sim}{F}^{2}$ & $\stackrel{1}{7}$ & $\stackrel{\vec{r}}{\vec{r}}$ & 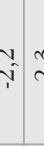 & ra & $\vec{i}$ & & & 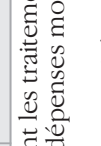 \\
\hline$\stackrel{\Xi}{\Xi}$ & 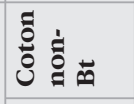 & & & & & & & & & $\hat{\tilde{\sigma}}$ & $\approx$ & ৫ & $\stackrel{\infty}{\stackrel{-}{-1}}$ & 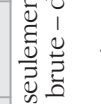 \\
\hline$\sum_{i}^{\infty}$ & قี & & & & & & & & & $\stackrel{+1}{ \pm}$ & 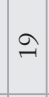 & 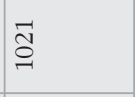 & సิ & 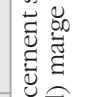 \\
\hline$\vec{E}$ & ڤే & & 華 & बे & 令 & $\stackrel{0}{\underset{7}{7}}$ & $\begin{array}{l}\Re \\
\stackrel{+}{+}\end{array}$ & 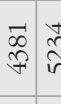 & \begin{tabular}{c|c}
0 \\
$\hat{n}$ \\
$n$
\end{tabular} & in & 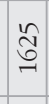 & $\underset{m}{\stackrel{m}{f}}$ & $\widetilde{\infty}$ & 递 \\
\hline 离 & ตั & 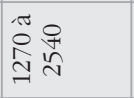 & 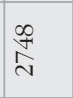 & 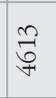 & $\begin{array}{l}\text { o } \\
\text { iै } \\
\text { in }\end{array}$ & $\begin{array}{l}0 \\
\vdots \\
\vdots \\
+1\end{array}$ & $\begin{array}{l}n \\
\stackrel{1}{ } \\
\infty \\
+\end{array}$ & 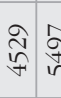 & 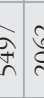 & ర్ & $\begin{array}{l}\alpha \\
\infty \\
\vec{N}\end{array}$ & \&̊ & f̂́ & 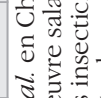 \\
\hline 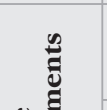 & ڤే & & & $\stackrel{\infty}{\infty}$ & $\hat{r}$ & $\hat{=}$ & $\tilde{6}$ & $\stackrel{\infty}{0} \stackrel{y}{r}$ & 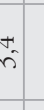 & & in & & & 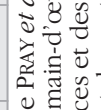 \\
\hline $\begin{array}{l}\tilde{y} \\
\hat{z} \\
\vec{z}\end{array}$ & ตี & & & 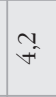 & in & $\stackrel{t}{r}$ & in & $\stackrel{\sim}{\sim}$ & F & & $\begin{array}{l}0 \\
i\end{array}$ & & & 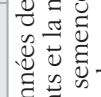 \\
\hline 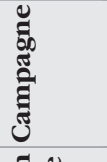 & & $\begin{array}{l}\sigma \\
\alpha \\
\alpha \\
\sigma \\
\sigma\end{array}$ & 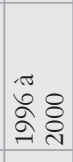 & $\begin{array}{l}\hat{\hat{o}} \\
\text { } \\
\text { ने }\end{array}$ & 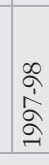 & 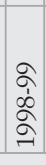 & 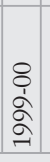 & 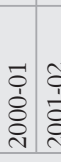 & 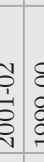 & 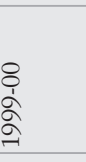 & 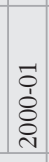 & 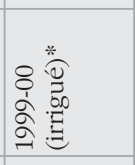 & 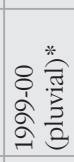 & 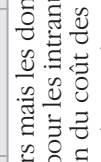 \\
\hline 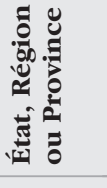 & & 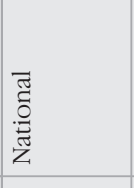 & 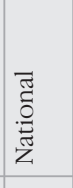 & 㺃 & & & & & & 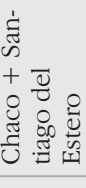 & & 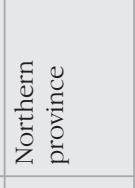 & 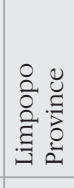 & 5 \\
\hline 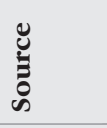 & & 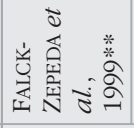 & 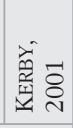 & E्ञ & & & & & & 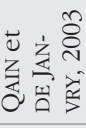 & & 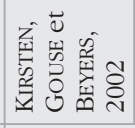 & & \\
\hline$\sum_{i}^{\infty}$ & & 峁 & & 总 & & & & & & 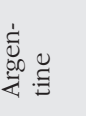 & & 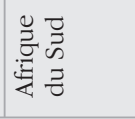 & & U \\
\hline
\end{tabular}


n'y a pratiquement pas de gain de rendement. La réduction du coût dans le contrôle des ravageurs est à peine perceptible (FITT, Wilson, MENSAH et DaLY, 2004). Aux États-Unis, les résultats (issus essentiellement des travaux d'expérimentation) sont très variés, car les conditions de pression parasitaire diffèrent entre les États. En moyenne, le gain de rendement serait de 3-11 \%, avec un niveau de rendement peu élevé. Les réductions des coûts de contrôle des ravageurs sont aussi de cet ordre (6-18\%). Globalement, le gain de productivité n'est pas élevé.

On peut même se demander si ce gain existe bien au regard des statistiques générales fournies par l'USDA ${ }^{4}$, et basées sur les éléments en conditions réelles de production. Par rapport à la période avant l'adoption du CGM, sur une période de dix ans après la commercialisation du CGM, le rendement moyen n'a été supérieur que lors des deux dernières années (figure 1). Les coûts de production à l'hectare ont augmenté car les coûts consacrés aux semences ont fortement augmenté, sans que ceux des pesticides (insecticides, herbicides, etc.) diminuent (figure 2). Le coût unitaire de production n'a réellement baissé que pour les deux dernières campagnes sur une période de dix ans (figure 3).

Figure 1 : Évolution du rendement du coton fibre aux États-Unis, 1975-2004

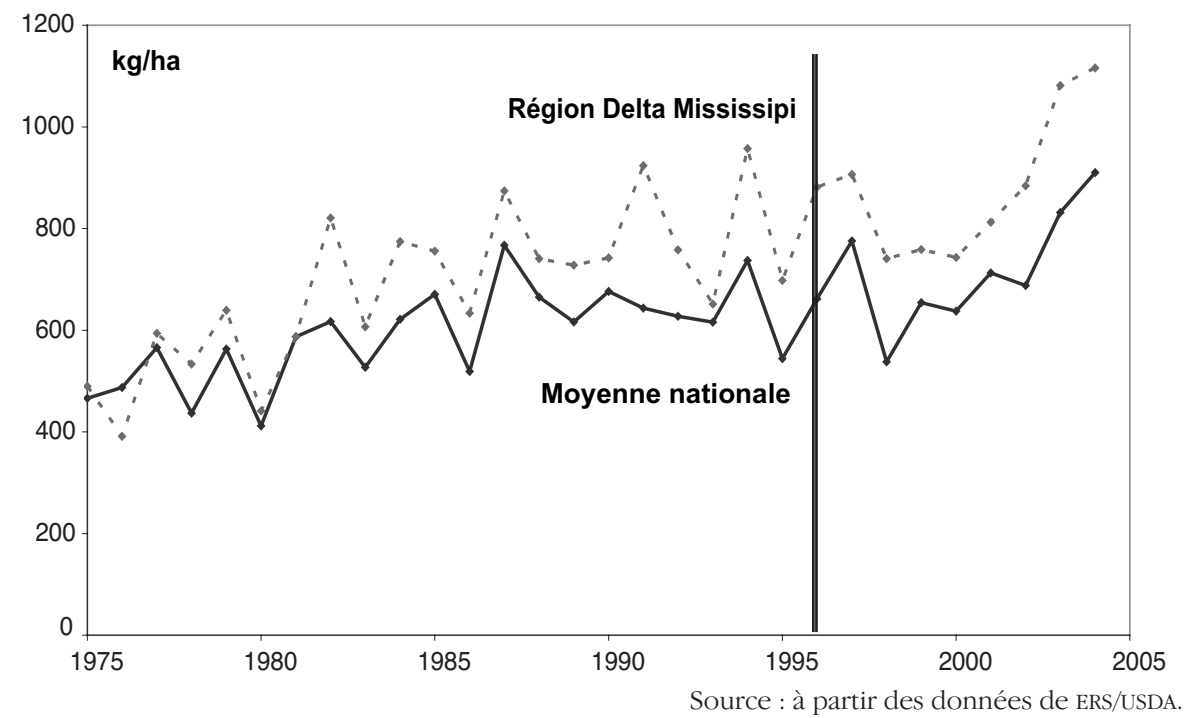

Note : À côté de la moyenne nationale, on a fait apparaître celle de la région du Delta de Mississipi où l'utilisation du coton Bt est plus forte.

Des enquêtes réalisées aux États-Unis, en Australie et auprès des fermiers blancs de l'Afrique du Sud, la notion de « confort » procuré par le coton Bt dans le contrôle des ravageurs ressort clairement. Les fermiers sont moins soumis à la contrainte du comptage des chenilles pour les traiter chimiquement aux stades où elles sont les plus sensibles. Le soulagement de cette contrainte est appréciable quand elle intervient à la période de Noël et des vacances d'été, comme c'est le cas

4 - The Economic Research Service de l'USDA fournit régulièrement des Farm Business Economics Report sur les principales productions agricoles du pays avec les statistiques sur les coûts de production. 
en hémisphère sud (Gouse, KIRSTEN et Beyers, 2002). En Australie, au «confort» mentionné s'ajoute la bonne conscience. Face à la pression de la société civile contre l'usage jugé excessif des pesticides, la réduction du nombre de traitements résultant du coton Bt permet aux planteurs de se sentir moins mal à l'aise face aux critiques écologistes (FITT, 2003).

Figure 2 : Évolution des coûts des semences et des pesticides aux États-Unis (Région Delta/Mississipi Portal), 1975-2004

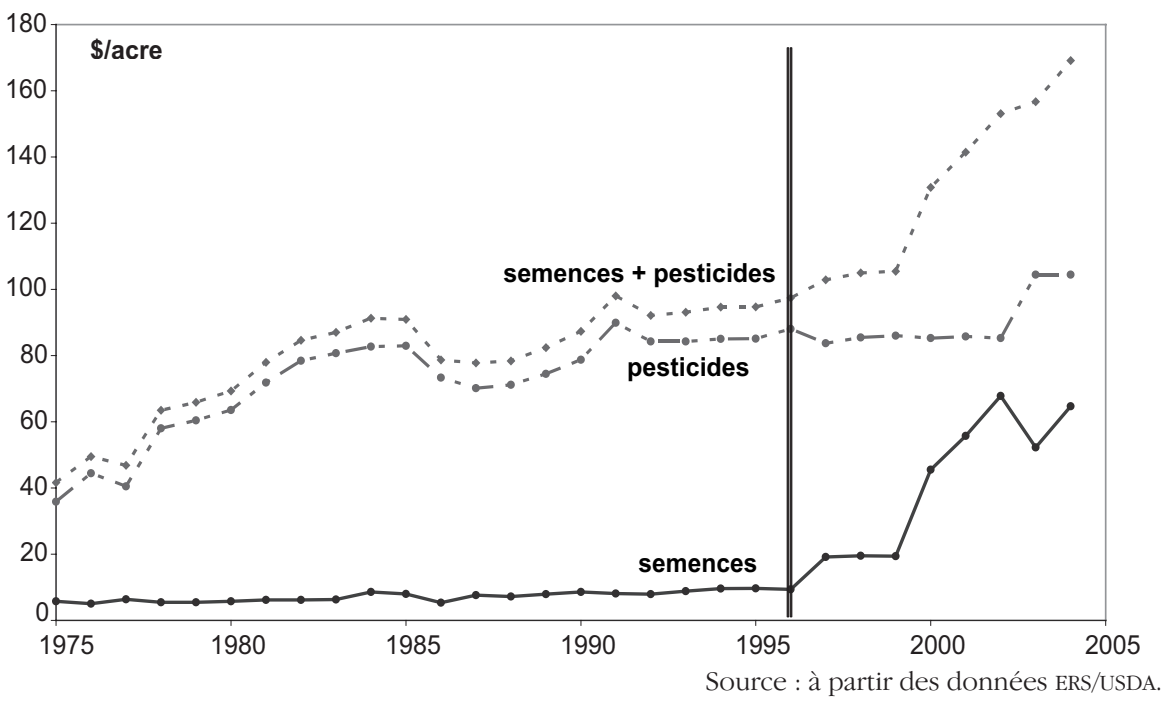

En production pluviale, les niveaux de rendement peuvent beaucoup varier entre les pays, en fonction des pluviométries mais aussi en fonction du degré d'intensification des cultures. Avec un niveau de rendement plus élevé, résultat d'une intensification plus poussée, la rentabilité à l'hectare est plus faible en Argentine qu'en Afrique du Sud. Cette rentabilité est aussi très dépendante d'une baisse du prix d'achat du coton produit (cas de l'année 2001). Dans ces conditions, l'intérêt économique du coton Bt est douteux si l'on se réfère aux valeurs de marges nettes (moins de $20 \$ /$ ha). L'indication d'un gain relatif important en marge nette du coton Bt (ici $58 \%$ ) peut être trompeuse, car le gain en valeur absolue peut être très faible.

\section{3 - Dans les petites exploitations, des effets variables aux facteurs multiples}

Dans les pays où le coton Bt est utilisé dans des exploitations familiales de petites tailles, les effets sont globalement positifs mais avec une grande variation du sens et de l'amplitude des effets observés. Une telle variation peut donner lieu à une grande divergence d'appréciation. Le cas le plus controversé concerne l'utilisation de semences hybrides de coton-Bt dans l'État d'Andhra Pradesh en Inde, réussite exemplaire pour certains - ISAAA $^{5}$, ICAC ${ }^{6}$, Monsanto, firmes

5 - International Service for the Acquisition of Agri-biotech Applications.

6 - International Cotton Advisory Committee. 
semencières internationales ou indiennes, mais aussi des économistes spécialisés dans le suivi des ogm (BennetT, IsmaËL, Kambhampati et Morse, 2004) -, échec cuisant pour d'autres - ONG indiennes et internationales relayées par les médias (DEVRAJ, 2006). Il doit néanmoins y avoir un problème : le Gouverneur de cet État vient de sommer la firme Mahyco ${ }^{7}$ (qui a fourni les semences) de dédommager les paysans (ANON, 2006b). Les causes possibles du problème sont cependant diverses (inefficacité des gènes utilisés, manque de formation et d'information des paysans, mauvaise qualité des semences de contrefaçon, etc.). Cet exemple témoigne que l'avantage économique de l'utilisation du coton Bt dans un PED ou un pays émergent est sous l'influence de facteurs autres que le caractère transgénique. On peut citer par exemple le facteur organisationnel dont on verra le rôle crucial dans la distribution des semences.

Figure 3 : Évolution du coût unitaire de production de coton fibre aux États-Unis, 1975-2004

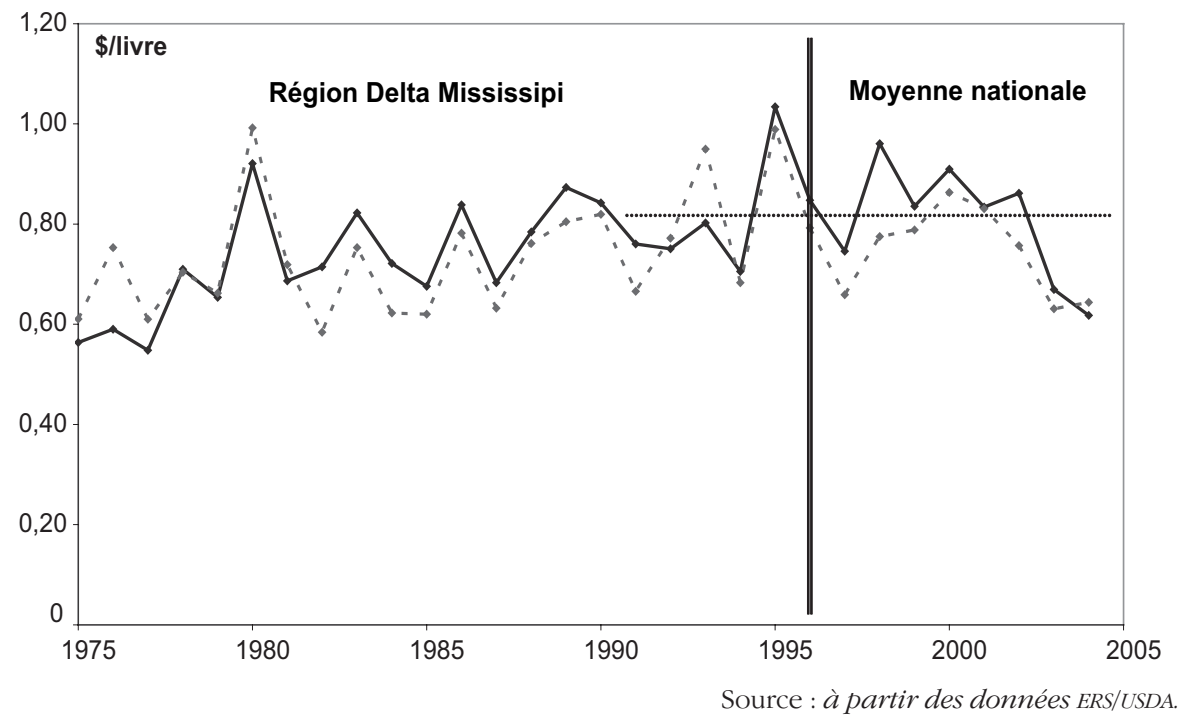

\section{a - La portée relative de l'efficacité et des externalités}

Partout où le coton Bt est utilisé, on observe une réduction du coût des insecticides utilisés pour contrôler les ravageurs du cotonnier (tableau 4). Cette réduction est variable selon les pays, selon les années dans un même pays, comme en Afrique du Sud (HoFs, FoK et VAISSAYRE, 2006), ou selon les régions d'un même pays, comme en Chine. Le degré de réduction dépend du niveau de pression parasitaire avant le recours au coton Bt et du nombre de traitements chimiques que les paysans appliquaient alors. Il en découle que la réduction du nombre de traitements, et donc celle du coût correspondant, est la plus forte en Chine, du moins dans les provinces situées le long de la vallée du Fleuve Jaune (HuANG, Hu, PraY, QiaO et ROZElle, 2003), mais pas dans tout le pays (Xu, You, WANG et YANG, 2004).

7 - Mahyco Monsanto Hybrid Company est une firme en joint venture associée à Monsanto. 


\begin{tabular}{|c|c|c|c|c|c|c|c|c|c|c|c|}
\hline \multirow{4}{*}{ 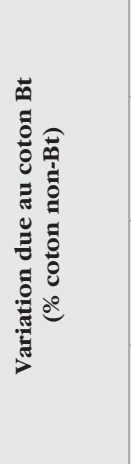 } & 总气 & वे & 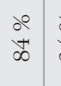 & \begin{tabular}{l|l}
20 & $\vdots$ \\
$⿱ ⺌$ & 1
\end{tabular} & $\stackrel{\circ}{\stackrel{2}{r}}$ & $\begin{array}{l}\stackrel{0}{0} \\
\stackrel{0}{0}\end{array}$ & 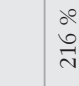 & $\stackrel{f}{\approx}$ & $\stackrel{\circ}{7}$ & $\begin{array}{l}\text { do } \\
i\end{array}$ & $\begin{array}{l}\stackrel{2}{2} \\
i f\end{array}$ \\
\hline & 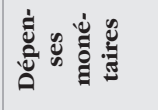 & $\begin{array}{l}\text { de } \\
\text { ते }\end{array}$ & $\stackrel{\circ}{\leftrightarrow}$ & $\begin{array}{l}\stackrel{0}{7} \\
7\end{array}$ & & $\stackrel{\circ}{\stackrel{2}{2}}$ & $\begin{array}{l}\stackrel{\circ}{\curvearrowright} \\
\stackrel{7}{\rightleftharpoons}\end{array}$ & & & & 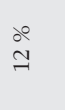 \\
\hline & 三气。 & & & & $\stackrel{\leftrightarrow}{\approx}$ & o̊ & 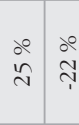 & $\begin{array}{l}\circ \\
\overrightarrow{i n}\end{array}$ & $\begin{array}{l}\stackrel{0}{0} \\
\underline{r} \\
-1\end{array}$ & 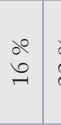 & $\begin{array}{l}\stackrel{\text { वे }}{\text { Uे }}\end{array}$ \\
\hline & 过 & $\therefore$ & $\begin{array}{l}\text { o } \\
\text { in }\end{array}$ & $\stackrel{\circ}{\Rightarrow}$ & $\stackrel{\circ}{0}$ & ळे & $\begin{array}{ll}\circ & \circ \\
i & 0 \\
\infty & i n\end{array}$ & $\begin{array}{l}\text { o̊ } \\
\text { ơ }\end{array}$ & $\begin{array}{l}\stackrel{\leftrightarrow}{\forall} \\
\forall\end{array}$ & $\stackrel{\circ}{\circ}$ & $\begin{array}{l}\stackrel{\circ}{2} \\
\ddot{\gamma}\end{array}$ \\
\hline 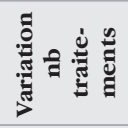 & & $\stackrel{\sim}{\overrightarrow{7}}$ & & & $\uparrow$ & & & & & & $\stackrel{0}{\circ}$ \\
\hline \multirow{2}{*}{ 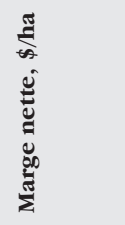 } & 递 & $\stackrel{F}{1}$ & $\begin{array}{l}\infty \\
\tilde{b}\end{array}$ & $\overrightarrow{\widetilde{\sigma}}$ & 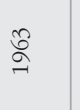 & $\vec{\infty}$ & 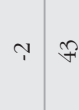 & $\stackrel{\infty}{\beth}$ & $\stackrel{\infty}{\dddot{J}}$ & ๑ & $i$ \\
\hline & ตี & $\hat{\swarrow}$ & 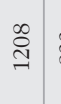 & $\approx$ & $\underset{\sim}{\stackrel{J}{\sim}}$ & $\stackrel{\hat{\sigma}}{\sigma}$ & in & $\stackrel{+}{\sim}$ & $\stackrel{\sim}{\sim}$ & $\stackrel{\curvearrowright}{\curvearrowleft}$ & $\infty$ \\
\hline \multirow{2}{*}{ 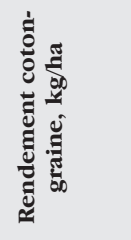 } & 泀 & $\begin{array}{l}\infty \\
\infty \\
\vec{m}\end{array}$ & $\vec{\Xi}$ & $\stackrel{\infty}{m}$ & $\begin{array}{l}\text { के } \\
\text { ळ. }\end{array}$ & $\tilde{w}$ & 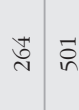 & nू & if & $\stackrel{+}{\stackrel{m}{*}}$ & $\underset{\exists}{\exists}$ \\
\hline & $\begin{array}{l}\ddot{\theta} \\
0 \\
0 \\
0\end{array}$ & $\underset{\aleph}{\stackrel{n}{\infty}}$ & 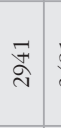 & 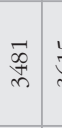 & $\frac{n}{\tilde{n}}$ & $\infty$ & $\underset{\stackrel{\alpha}{\alpha}}{\stackrel{\infty}{\wedge}}$ & $\stackrel{0}{i}$ & 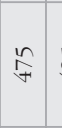 & $\approx$ & กิ \\
\hline \multirow{2}{*}{ 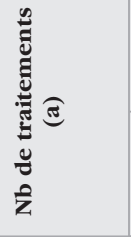 } & ㅎํㅎํ & $\stackrel{\infty}{\stackrel{2}{q}}$ & & & $a$ & & & & & & $\stackrel{n}{i}$ \\
\hline & $\begin{array}{l}\vec{a} \\
\vdots \\
\vdots \\
0\end{array}$ & $\sigma_{0}^{0}$ & & & $r$ & & & & & & $\Rightarrow$ \\
\hline \multicolumn{2}{|l|}{ 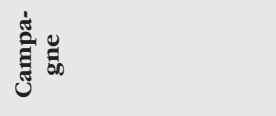 } & $\begin{array}{l}8 \\
\stackrel{1}{\alpha} \\
\text { ने }\end{array}$ & \begin{tabular}{l|l} 
& \\
0 & \\
$\vdots$ & \\
8 &
\end{tabular} & 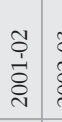 & 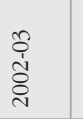 & $\begin{array}{l}\alpha \\
\alpha \\
\alpha \\
\curvearrowright\end{array}$ & 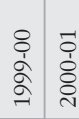 & $\begin{array}{l}8 \\
\vdots \\
\vdots \\
2 \\
2\end{array}$ & $\begin{array}{l}\stackrel{\alpha}{\alpha} \\
\stackrel{\alpha}{\alpha}\end{array}$ & 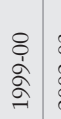 & 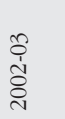 \\
\hline \multicolumn{2}{|l|}{ 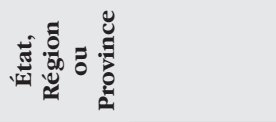 } & \multicolumn{3}{|c|}{ 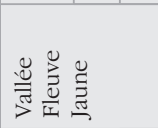 } & 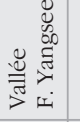 & \multicolumn{2}{|l|}{ 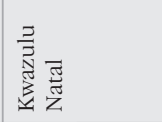 } & 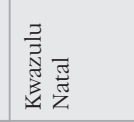 & \multicolumn{2}{|l|}{ 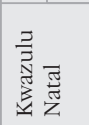 } & 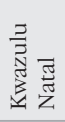 \\
\hline \multicolumn{2}{|l|}{ 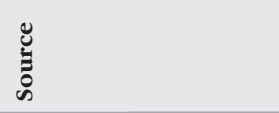 } & 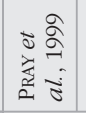 & 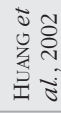 & & 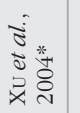 & \multicolumn{2}{|c|}{ 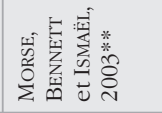 } & 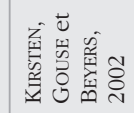 & \multicolumn{2}{|c|}{ 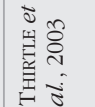 } & 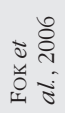 \\
\hline \multicolumn{2}{|l|}{$\stackrel{n}{E}$} & \multicolumn{4}{|l|}{ 气̆ } & \multicolumn{6}{|l|}{ 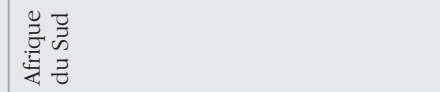 } \\
\hline
\end{tabular}




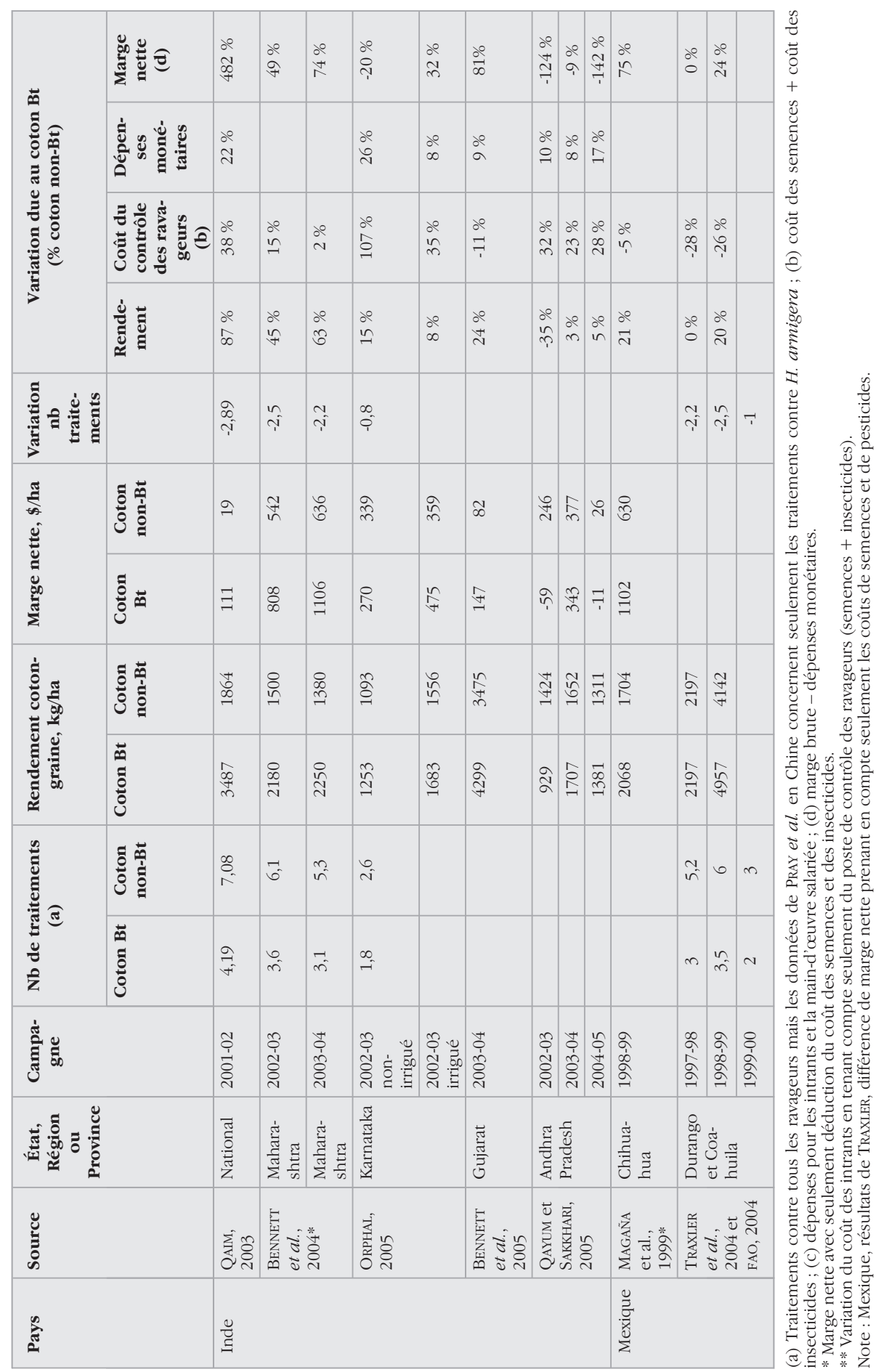


Dans aucun pays les traitements insecticides n'ont disparu. Les toxines produites par le coton Bt ne sont efficaces que contre certains types de ravageurs cibles (les chenilles de lépidoptères, surtout Helicoverpa armigera mais aussi Pectinophora gossypiella, Earias sp.), pendant une partie du cycle du cotonnier et si les conditions climatiques ne nuisent pas à l'expression du gène Bt. Il reste donc nécessaire de traiter, un peu, contre les ravageurs cibles. Il est aussi normal de constater que le nombre de traitements chimiques contre les ravageurs non-cibles du coton Bt ne diminue pas. Ce nombre peut même augmenter, comme en Afrique du Sud et surtout en Chine (FOK, LIANG, WANG et WU, 2005 ; LANG, 2006).

La réduction du coût total de protection phytosanitaire, résultant du coût des insecticides et des semences de coton Bt, est loin d'être systématique. Ce coût total est même fréquemment plus élevé avec le coton Bt, car le surcoût des semences n'est pas compensé par l'économie découlant de la diminution du nombre de traitements chimiques.

La réduction du nombre de traitements insecticides est généralement associée à un effet bénéfique pour la santé des paysans. Cette perception est à nuancer (Hofs, FoK et VAISSAYRe, 2006 ; BenneTt, Ismä̈L et MORSE, 2004). Les produits insecticides que le coton Bt permet d'économiser (les pyréthrinoïdes) ont une toxicité faible par rapport aux produits organophosphorés qu'il faut utiliser, parfois en plus grande quantité, pour traiter contre les ravageurs non-cibles.

La réduction du nombre de traitements insecticides par le coton Bt se traduit par une diminution du travail et de la pénibilité du travail associé. L'ampleur de cette réduction est cependant souvent exagérée. Pour la Chine, HuANG et al. signalent une économie en travail de plus de 80 jours/ha (HuANG, Hu, PRAY, QIAO et RozELLE, 2003) mais c'est en retenant une hypothèse irréaliste d'un travail de cinq jours pour traiter un hectare de coton.

S'agissant du coton Bt, il est important de rappeler que l'effet positif sur le rendement n'était pas avancé lors de son lancement en 1996. L'intérêt anticipé par les promoteurs de ce coton était la diminution du coût de production par la réduction du nombre de traitements insecticides. L'augmentation du rendement observé dans certains cas était donc une bonne surprise. Cette augmentation est cependant variable selon les pays et les années. Pour l'Afrique du Sud, les gains très importants observés dans les premiers travaux (ThIRTLE, JENKINS BEYERS, 2003) n'ont pas été confirmés dans les travaux plus récents (HOFS, FOK, GOUSE et KiRSTEN, 2006 ; HoFs, FoK et VAISSAYRe, 2006). C'est dans les petites exploitations des PED que le gain de rendement observé est le plus élevé, en valeur relative. Il y a en fait une relation inverse entre le niveau initial et le gain (relatif) de rendement. C'est quand le niveau initial est faible que le taux de gain est plus élevé. Ce point est intéressant et il est favorable aux paysans dont le niveau de rendement est pénalisé par diverses contraintes structurelles de leurs exploitations.

Le rendement du cotonnier dépend du contrôle des ravageurs. Globalement, l'absence de traitements chimiques fait perdre $35-41 \%$ du rendement potentiel, mais il y a une perte de rendement de 7-24 \% même quand le contrôle chimique des ravageurs est appliqué (ISAAA, 2005). Dans la réalité, il est techniquement impossible de traiter systématiquement à meilleur escient, au moment où les 
chenilles sont aux stades les plus sensibles, et de manière à les atteindre partout où elles peuvent se nicher. À titre indicatif, les produits chimiques peuvent être lavés par les pluies qui surviennent après leur épandage.

Les traitements chimiques ne sont donc pas parfaitement efficaces et ne peuvent pas l'être. Il y a forcément un manque à gagner par rapport au rendement potentiel et ce manque est d'autant plus grand que le contrôle chimique est insuffisant ou mal réalisé. L'utilisation du coton Bt permet en fait de récupérer partiellement le manque à gagner et de s'approcher davantage du rendement potentiel. Dans beaucoup de PED, il y a de nombreuses raisons qui empêchent la réalisation correcte de la protection chimique et que les paysans ne peuvent pas contrôler (absence de crédit, défaillances du réseau de distribution des intrants, etc.). L'utilisation du coton Bt réduit ainsi la dépendance des paysans à l'égard des imperfections de ce type de contexte et cela constitue indéniablement un élément positif.

\section{b - Une rentabilité variable, sous dépendance}

Le gain de rentabilité procuré par l'utilisation du coton Bt est variable et il dépend fondamentalement de quatre facteurs : le surcoût des semences, le degré de réduction du coût des insecticides, le degré de gain de rendement, le niveau et la stabilité du prix de vente du coton-graine. Le premier facteur est le seul qui dépende des conditions de distribution des semences de coton $\mathrm{Bt}$, mais il focalise la méfiance des opposants aux OGM. Les autres facteurs sont dépendants des conditions des pays utilisateurs et méritent aussi d'être pris en compte dans les réflexions sur les conditions de rentabilité du coton Bt.

Lorsque le surcoût des semences n'est pas trop élevé et qu'il est facilement compensé par la réduction du coût des insecticides, qu'un gain de rendement est enregistré, avec un produit qui ne souffre pas d'une décote de prix, on a la situation idéale d'un gain de rentabilité indéniable. Une telle combinaison favorable des quatre facteurs est cependant assez rare. La Chine bénéficie quasiment de cette combinaison favorable mais, dans beaucoup d'autres PED, le gain de rentabilité n'est pas si élevé parce que le coût du contrôle des ravageurs n’a pas diminué, quand il n'a pas augmenté.

Ces observations justifient la méfiance des opposants aux OGM car la cherté des semences pénalise les paysans utilisateurs. Cette cherté pénalise aussi les fournisseurs de semences car l'extension de l'utilisation du CGM s'en trouve limitée (QAIM et DE JANVRY, 2003) et que les utilisateurs sont encouragés à tricher pour ne pas payer la redevance d'emploi jugée trop élevée (Trigo, CHuDNOvsKY, CAP et LOPEZ, 2003).

Dans une année donnée, au niveau d'un paysan, l'espérance de la rentabilité du coton Bt dépend de celle du rendement. Or l'espérance de rendement en production pluviale et en petit paysannat peut être limitée pour des raisons incontrôlables, du domaine climatique (installation tardive des pluies par exemple), du domaine institutionnel (approvisionnement défaillant en semences ou en intrants dans une filière déstabilisée), ou pour des raisons propres à l'exploitation (manque de main-d'œuvre). Cela est vrai même sans le recours aux CGM mais, avec 
l'utilisation de semences CGM nécessitant un débours monétaire important en début de saison, l'incidence négative sur la rentabilité est plus forte.

Cette analyse met en évidence le danger économique à prôner l'utilisation exclusive du CGM, comme cela est implicite dans le débat actuel.

\section{III - LES ÉLÉMENTS À GÉRER POUR UNE EXTENSION BÉNÉFIQUE DU COTON BT DANS LES PED}

Le débat sur la pertinence des OGM pour les PED oppose des effets anticipés par les partisans et les opposants que l'on peut résumer dans le cas du coton $\mathrm{Bt}$ (tableau 5).

Tableau 5 : Synthèse sur les effets anticipés relatifs à l'utilisation du coton Bt

\begin{tabular}{|l|l|}
\hline \multicolumn{1}{|c|}{ Effets positifs } & \multicolumn{1}{|c|}{ Effets négatifs } \\
\hline $\begin{array}{l}\text { Coton Bt efficace contre les ravageurs } \\
\text { cibles }\end{array}$ & Non-efficacité contre les ravageurs cibles \\
\hline Réduction de l'usage des insecticides & $\begin{array}{l}\text { Coût élevé des semences pour les utilisa- } \\
\text { teurs }\end{array}$ \\
\hline $\begin{array}{l}\text { Réduction du coût de contrôle des rava- } \\
\text { geurs }\end{array}$ & $\begin{array}{l}\text { Effet de barrière à l'utilisation de semences } \\
\text { pour beaucoup de paysans }\end{array}$ \\
\hline Rentabilité améliorée & $\begin{array}{l}\text { Instauration d'une situation de monopole } \\
\text { des semences de coton Bt }\end{array}$ \\
\hline Contribution à la réduction de la pauvreté & $\begin{array}{l}\text { Obligation du renouvellement annuel des } \\
\text { semences qui accentue les coûts de pro- } \\
\text { duction }\end{array}$ \\
\hline Réduction du travail et de sa pénibilité & Effets néfastes sur la faune non-cible \\
\hline Effet positif sur la santé & $\begin{array}{l}\text { Induction d'une résistance des ravageurs à } \\
\text { la toxine Bt }\end{array}$ \\
\hline & $\begin{array}{l}\text { Impossibilité pour le coton non GM de } \\
\text { coexister avec le coton Bt }\end{array}$ \\
\hline & $\begin{array}{l}\text { Diffusion incontrôlée du gène Bt avec des } \\
\text { effets indésirables }\end{array}$ \\
\hline &
\end{tabular}

À la lumière des résultats rapportés ci-dessus, on peut donner une vision plus complète des effets de l'utilisation du coton Bt. Les effets anticipés ne sont pas tous confirmés ou de manière systématique (tableau 6). 
Tableau 6 : Des effets de 1'utilisation du coton Bt imparfaitement anticipés

\begin{tabular}{|l|l|l|}
\hline \multicolumn{1}{|c|}{ Effets positifs } & \multicolumn{1}{|c|}{ Effets neutres } & \multicolumn{1}{|c|}{ Effets négatifs } \\
\hline $\begin{array}{l}\text { Efficacité avérée du coton } \\
\begin{array}{l}\text { Bt contre les ravageurs } \\
\text { cibles }\end{array}\end{array}$ & $\begin{array}{l}\text { Nuisance non établie sur la } \\
\text { faune non-cible }\end{array}$ & $\begin{array}{l}\text { Instauration } \\
\text { d'une situation de mono- } \\
\text { pole pour les semences de } \\
\text { CGM }\end{array}$ \\
\hline $\begin{array}{l}\text { Réduction avérée de de } \\
\text { l'usage des insecticides } \\
\text { contre les ravageurs cibles, } \\
\text { mais réduction seulement }\end{array}$ & $\begin{array}{l}\text { Nuisance non-établie sur les } \\
\text { prédateurs des ravageurs }\end{array}$ & $\begin{array}{l}\text { Chamboulement des servi- } \\
\text { ces semenciers établis }\end{array}$ \\
\hline $\begin{array}{l}\text { Réduction du coût des } \\
\text { insecticides, mais réduc- } \\
\text { tion seulement }\end{array}$ & $\begin{array}{l}\text { Émergence non avérée de } \\
\text { résistance des ravageurs } \\
\text { cibles aux toxines Bt }\end{array}$ & $\begin{array}{l}\text { Obligation quasi systémati- } \\
\text { que du renouvellement } \\
\text { annuel des semences }\end{array}$ \\
\hline $\begin{array}{l}\text { Rentabilité fréquemment } \\
\text { améliorée mais à des } \\
\text { degrés très variables }\end{array}$ & $\begin{array}{l}\text { Peu d'éléments pour statuer } \\
\text { sur la coexistence (CGM et } \\
\text { coton conventionnel) }\end{array}$ & $\begin{array}{l}\text { Coût élevé des semences de } \\
\text { manière très fréquente }\end{array}$ \\
\hline \multicolumn{2}{|c|}{} & $\begin{array}{l}\text { Réduction exceptionnelle } \\
\text { du coût total du contrôle des } \\
\text { ravageurs }\end{array}$ \\
\hline
\end{tabular}

Il y a aussi un grand nombre d'effets non réellement anticipés, qui peuvent d'ailleurs être positifs ou négatifs (tableau 7). Il ressort que, même avec l'attention que le sujet avait attirée, l'anticipation des effets a été très incomplète, malgré les connaissances disponibles.

On observe un excès de pessimisme ou d'optimisme dans les deux catégories d'effets, comme cela ressort de l'analyse qui suit.

\section{1 - Un optimisme et un pessimisme excessifs}

Les effets anticipés, positifs ou négatifs, ne sont pas confirmés de manière systématique (tableau 6). On constate aussi que l'anticipation a été très incomplète car de nombreux effets n'avaient pas été pressentis (tableau 7). Il ressort un continuum d'effets, mal anticipés, allant de bonnes surprises (des effets positifs non prévus) à des menaces non soupçonnées (effets négatifs non prévus), en passant par des effets anticipés plus ou moins confirmés.

S'agissant des effets anticipés initialement, force est de constater qu'il n'y pas de confirmation complète et systématique, que les effets soient positifs ou négatifs. Les effets anticipés non avérés forment ainsi un troisième groupe d'effets neutres.

S'agissant des effets positifs anticipés, l'efficacité du coton Bt contre les ravageurs qu'il est censé contrôler est peu contestable. Elle se traduit par la réduction du nombre de traitements insecticides, de l'ordre de 2 à 3. Les traitements 
Tableau 7 : Des effets de l'utilisation du coton Bt incomplètement anticipés

\begin{tabular}{|l|l|}
\hline \multicolumn{1}{|c|}{ Effets positifs } & \multicolumn{1}{|c|}{ Effets négatifs } \\
\hline $\begin{array}{l}\text { Gain de rendement, mais à un degré varia- } \\
\text { ble }\end{array}$ & $\begin{array}{l}\text { Changement de la structure des coûts de } \\
\text { production accentuant le risque financier } \\
\text { de l'utilisation des semences de cGM }\end{array}$ \\
\hline $\begin{array}{l}\text { «Confort » dans la réalisation du contrôle } \\
\text { contre les ravageurs }\end{array}$ & $\begin{array}{l}\text { Effets pervers des comportements oppor- } \\
\text { tunistes pour ne pas payer la redevance } \\
\text { d'emploi des semences }\end{array}$ \\
\hline $\begin{array}{l}\text { Affranchissement des incertitudes } \\
\text { d'obtention des insecticides pour les pay- } \\
\text { sans dans de nombreux PED }\end{array}$ & $\begin{array}{l}\text { Accentuation du coût des semences pour } \\
\text { des changements de conditions de distri- } \\
\text { bution des semences pour mieux préserver } \\
\text { la rente biotechnologique }\end{array}$ \\
\hline $\begin{array}{l}\text { Retour possible à un niveau bien plus faci- } \\
\text { lement gérable de la pression parasitaire }\end{array}$ & $\begin{array}{l}\text { Grande variation de la rentabilité entre les } \\
\text { pays, entre les régions d'un pays, entre les } \\
\text { années, et entre les producteurs }\end{array}$ \\
\hline $\begin{array}{l}\text { Capacité, quoique rare, des utilisateurs à } \\
\text { intégrer le coton Bt dans une approche } \\
\text { intégrée de contrôle des ravageurs }\end{array}$ & $\begin{array}{l}\text { Danger économique de la couverture à } \\
100 \% \text { en cGM }\end{array}$ \\
\hline $\begin{array}{l}\text { Il y a une marge pour la négociation des } \\
\text { conditions de distribution et d'utilisation } \\
\text { des semences de cGm }\end{array}$ & $\begin{array}{l}\text { Danger écologique de la couverture à } \\
100 \% \text { en cGM }\end{array}$ \\
\hline $\begin{array}{l}\text { Il y a une marge pour la négociation du } \\
\text { coût d'utilisation des semences de cGM }\end{array}$ & $\begin{array}{l}\text { Changement défavorable de la composi- } \\
\text { tion du complexe parasitaire }\end{array}$ \\
\hline
\end{tabular}

insecticides restent nécessaires, pour contrôler les ravageurs cibles du coton Bt et les ravageurs non-cibles. Il n'y a qu'une réduction du nombre de traitements chimiques sans forcément induire une diminution du coût total du contrôle (quand on combine le coût des semences et celui des traitements insecticides). Il en découle que l'amélioration de la rentabilité du coton Bt procède beaucoup du gain de rendement, plus fréquemment observé dans les PED, mais elle est souvent assez faible et fragile.

Cela est essentiellement dû à l'effet négatif d'une augmentation forte et générale du coût des semences. Comme cela a été craint, l'instauration d'un monopole dans la distribution des semences de coton Bt est quasi systématique, la Chine est une exception. Une telle situation de monopole a aussi provoqué l'effondrement du système semencier préexistant (TRIGO, CHUDNOvsKY, CAP et LOPEZ, 2003).

Par contre, il n'y a pas d'éléments pour justifier les craintes relatives aux impacts sur la faune non-cible. Il n'y a pas non plus de cas avérés d'apparition de résistance des ravageurs cibles contre la toxine Bt. On ne peut pas encore se 
prononcer sur l'impossibilité de faire du coton conventionnel dans les zones ayant adopté le coton Bt.

\section{2 - Les avantages d'une certaine flexibilité, méconnue}

La colonne de gauche du tableau 7 indique que plusieurs effets positifs ne furent pas explicitement anticipés. Il n'est pas nécessaire de s'appesantir sur le gain de rendement, plus important, en termes relatifs, pour les paysans souffrant d'un niveau de rendement plus faible.

Tous les autres effets positifs non réellement anticipés révèlent un avantage de flexibilité procurée par le coton $\mathrm{Bt}$, notamment dans la pratique même du contrôle chimique des ravageurs, pour les grandes comme pour les petites exploitations.

Plus intéressante à souligner est la réversibilité des équilibres biologiques. En quelques années l'utilisation du coton Bt permet de revenir à un niveau de pression parasitaire plus faible et beaucoup plus facile à gérer, surtout en Chine où cette pression était parvenue à un niveau très élevé (FOK, LIANG, WANG et Wu, 2005). Bien sûr, un tel retour à une situation favorable peut ne pas durer si on n'y prend garde (cf. infra), mais l'observation de cette réversibilité est riche en enseignements. L'hypothèse implicite sur l'occurrence brutale et irréversible des impacts environnementaux négatifs paraît ainsi discutable. Cela peut conduire à revoir les démarches actuelles de précaution, dont les coûts afférents (en temps et en termes financiers), notamment dans la conduite d'expérimentations de biosécurité, peuvent être jugés excessifs et insensés, et surtout pénalisants pour les PED (Pray, Bengali et Ramaswami, 2005).

Il faudrait peut-être oser manifester un peu plus de confiance dans la capacité de réaction des paysans qui, face à des signes d'évolution indésirable des équilibres biologiques, sont capables de changer de pratiques. Le cas des fermiers australiens réussissant à intégrer l'utilisation du coton Bt dans une démarche de lutte intégrée (FITT, WILSON, MENSAH et DALY, 2004) est un témoignage positif dans ce sens. Certes, il serait hasardeux d'extrapoler l'acquis des 1200 fermiers australiens au grand nombre de paysans cotonniers des PED. C'est cependant une leçon intéressante pour aider les PED à se prémunir contre les messages qui tendent soit à encenser soit à diaboliser l'utilisation du coton Bt.

Enfin, un autre point positif se rapporte à une certaine variation des conditions d'utilisation du coton Bt. Tous les pays n'ont pas eu à se plier aux conditions d'utilisation imposées par Monsanto : en termes d'engagement par contrat explicite et individuel, en termes de paiement de redevance d'emploi ou encore en termes d'acceptation sans négociation du niveau de cette redevance. La bonne nouvelle est donc qu'il existe un espace de négociation possible sur les conditions d'utilisation du coton Bt.

Fondamentalement c'est ce niveau de redevance qui détermine, pour beaucoup de paysans, la rentabilité de l'utilisation du coton Bt. Il doit donc être négocié en fonction des espérances de gain que cette utilisation peut procurer. Plus on admet que les espérances de gain sont élevées, plus il faudra s'attendre à payer une 
redevance élevée. La surestimation de l'espérance est donc une bonne pratique commerciale, pour le commerçant. Quant au client, il doit prendre garde à une surestimation délibérée et infondée de l'espérance de gain.

\section{3 - D'importants risques immédiats et dans futur proche}

La colonne de droite du tableau 7 met en évidence plusieurs niveaux de risque qui n’ont pas été explicités.

Le surcoût important des semences est porteur de risque financier élevé. Il se traduit par un changement de la structure des coûts de production (HoFs, FOK, GOUSE et KIRSTEN, 2006) avec une forte augmentation de la part des dépenses à consentir dès l'installation de la culture. La perception du risque financier est ainsi accrue pour les paysans à ressources financières limitées. Dans cette situation, les paysans ont deux options de réaction : ne pas adopter ou adopter la technique en trouvant des moyens pour réduire le coût du coton Bt. Dans ce deuxième cas, une manière de faire est de réduire les doses d'utilisation. Une façon moins légale est de chercher à payer moins cher les semences, en puisant sur sa propre production même si on n'en a pas le droit, ou en achetant des semences illégales...

Ces comportements opportunistes peuvent induire des conséquences néfastes, pour les individus concernés, mais pas seulement. La détermination du surcoût des semences doit donc intégrer la capacité de contournement des paysans dans les PED et éviter que la «redevance ne tue la redevance » en étant fixée à un niveau jugé inacceptable. Fixer ce niveau en fonction de l'espérance de gain des producteurs les moins performants serait une grosse erreur.

\section{a - Les dangers d'une couverture à $100 \%$ en coton $\mathrm{Bt}$}

La grande dispersion du rendement est un fait établi dans la production agricole familiale des PED car beaucoup de facteurs empêchent les paysans de s'installer et d'entretenir leurs cultures de manière optimale. Chaque année, l'utilisation du coton Bt est forcément non rentable pour une partie des parcelles ou des exploitations souffrant de rendements insuffisants.

En d'autres termes, il y a forcément une partie des parcelles qu'il ne faudrait pas faire ensemencer en coton Bt. Ce ne seront pas forcément les mêmes parcelles d'une année à l'autre, ni celles des mêmes paysans. Même si un PED a décidé d'adopter le coton $\mathrm{Bt}$, la cohabitation avec du coton conventionnel est économiquement justifiée. La flexibilité doit être préservée pour que chaque paysan puisse décider, pour chacune de ses parcelles, d'utiliser ou non le coton Bt, en fonction des conditions climatiques et de ses contraintes personnelles. Pour que les paysans puissent appliquer cette flexibilité au mieux de leurs intérêts, ils doivent être informés sur les facteurs déterminant la rentabilité du coton Bt. À défaut, les résultats divergents obtenus par les paysans les amèneront à s'opposer dans la perception de l'intérêt du coton Bt, à remettre en cause la décision relative à l'adoption de ce dernier et à douter de l'intégrité morale des décideurs.

Le raisonnement développé ici, en faveur d'une flexibilité à accorder aux paysans, s'oppose ainsi à l'hypothèse implicite d'une couverture à 100 \% en coton 
$\mathrm{Bt}^{8}$, hypothèse tenue tant par les fournisseurs de coton Bt que par les opposants aux OGM (pour la combattre). Sur le plan économique, l'abandon de cette hypothèse implicite aiderait au débat sur les OGM, tout en posant un délicat problème de coordination dans une utilisation partielle.

La couverture à $100 \%$ en coton Bt n'est pas non plus justifiable sur le plan écologique. Le retour à un plus faible niveau de pression parasitaire, quelques années après l'utilisation du coton Bt, est un acquis positif mais fragile. Le souci de la préservation de ce faible niveau retrouvé s'oppose à une couverture à $100 \%$ en coton Bt qui accentue le risque de voir émerger effectivement la résistance des ravageurs aux toxines Bt.

La focalisation sur ce dernier risque paraît excessive. Elle est aussi trompeuse dans la mesure où elle a détourné l'attention sur les risques plus importants liés aux changements du complexe parasitaire. Dans ce domaine, il n'est pas seulement question de changement d'équilibre entre les familles de ravageurs, avec une importance accrue des insectes piqueurs-suceurs par exemple (HoFs, FoK et VAISSAYRE, 2006). Le changement intervenant au sein des ravageurs d'une même famille est déjà observé, en Amérique latine et en Chine, et il paraît fort inquiétant. La chenille, Spodoptera litura (en Asie) ou litoralis (en Afrique et en Amérique), fort vorace, ne se contente plus de manger seulement les feuilles de cotonnier. Elle s'attaque aux organes fructifères (LI, 2004) sans doute parce qu'il y a moins de chenilles de Helicoverpa armigera, très agressives, pour les en empêcher !

\section{b - L'organisation de la distribution des semences : des ajustements importants sont nécessaires}

L'adoption du coton Bt suivant une démarche de couverture partielle impose la nécessité d'une organisation adaptée de la distribution des semences. Dans les PED, comme c'est le cas dans tous les pays au Sud du Sahara, la distribution des semences dans les villages est réalisée plusieurs mois avant le semis et avec une seule variété par village. Le principe de couverture partielle en coton Bt amène à réviser les modalités de cette distribution de semences. De nouvelles modalités efficaces ne paraissent pas évidentes à mettre en œuvre car les parts relatives du coton $\mathrm{Bt}$ et du coton conventionnel, à placer, dépendent notamment du facteur climatique et ne peuvent être déterminées parfaitement à l'avance. Pour éviter la conséquence fâcheuse d'une hétérogénéité de la qualité du coton fibre, il faudrait que les variétés de coton Bt et de coton conventionnel soient isogéniques (ne se différenciant que sur le seul caractère Bt).

Si cette réorganisation n'est pas mise en place, on peut anticiper que les paysans s'arrangeront par eux-mêmes, en catimini, induisant ainsi mélange variétal et hétérogénéité de la qualité du produit final ${ }^{9}$. On peut alors craindre que les

8 - À la fraction des parcelles refuges près, là où l'installation de ces parcelles est de mise.

9 - En Chine, la diffusion du CGM est responsable d'une multiplicité de variétés cultivées dans un même village, induisant un problème reconnu d'hétérogénéité de la qualité du coton produit. 
impacts négatifs pour les paysans et les filières concernées soient bien plus importants que les gains économiques espérés.

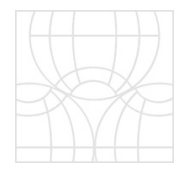

Dans le débat sur les ogm, que d'aucuns trouvent futiles (ELLIESEN, 2003), omissions, demi mensonges ou demi vérités et extrapolations abusives sont monnaies courantes. S'y glissent même quelques cas de corruption ${ }^{10}$ (GALA, 2005). L'interrogation sur la rigueur et l'intégrité morale des scientifiques qui vantent les mérites du coton Bt n'est pas sans fondement non plus (RAO, 2003), et cette interrogation peut être étendue aux journaux scientifiques se livrant à la course à la publication des résultats relatifs au succès des OGM.

L'analyse réalisée s'est concentrée sur le cas du coton Bt. Elle indique que, à part la confirmation d'un surcoût élevé des semences dans tous les pays utilisateurs, il y a fréquemment exagération sur les effets anticipés, qu'ils soient positifs ou négatifs.

L'utilisation du coton Bt n'affranchit pas des traitements chimiques et ne peut pas en affranchir totalement. Cette utilisation ne réduit pas forcément les coûts de contrôle des ravageurs. Les conditions d'utilisation, techniques et économiques, présentent un peu plus de diversité qu'initialement évoquée.

Les impacts observés du coton Bt diffèrent entre les pays et les modes de production. Dans les pays développés, l'utilisation de ce coton dans les grandes exploitations ne dégage pas un très net intérêt économique, mais le gain vient surtout du sentiment de « confort » et de bonne conscience dans le contrôle des ravageurs.

Dans les PED, l'efficacité du coton Bt contre les ravageurs cibles est indéniable en permettant d'économiser 2 à 3 traitements chimiques. Le gain de rendement est aussi plus fréquent et plus net. Sur le plan technique, les perspectives de l'extension de l'utilisation de ce coton paraissent bonnes. Sur le plan économique, l'appréciation doit être plus nuancée. Il est rare de constater une réduction du coût total du contrôle des ravageurs parce que le surcoût des semences dépasse l'économie réalisée sur les insecticides. Globalement, c'est le gain de rendement qui permet de parvenir in fine à une rentabilité améliorée, au prix d'une perception accrue du risque financier. La sécurité économique dans l'utilisation du coton Bt dépend fondamentalement du coût des semences et des conditions économiques d'utilisation pour lesquels les pays disposent d'une marge de négociation, à condition de savoir tirer profit des acquis d'autres pays. En Inde, le prix des semences vient de baisser radicalement ${ }^{11}$, c'est fondamentalement le résultat de

10 - En 2005, Monsanto a été condamné en Indonésie pour corruption de fonctionnaires dans les procédures de biosécurité.

11 - Depuis juin 2006, le paquet de $450 \mathrm{~g}$ de semences hybrides de coton Bt en Inde est passé de 1650-1700Rs à 750Rs. 
l'intervention des pouvoirs publics, rendue sans doute nécessaire par l'implication de la société civile. Il y a un espace de négociation sur le coût des semences de CGM, il serait regrettable que les pouvoirs publics des PED continuent à l'ignorer et à occulter le rôle qu'ils peuvent jouer.

Avec le recul permis par dix ans d'utilisation du coton $\mathrm{Bt}$, on constate que les anticipations sur les effets ont été imparfaites, comme on vient de le voir, et surtout très incomplètes. En quelques années, l'utilisation du coton Bt permet de ramener la pression parasitaire à un niveau plus faible et plus facile à gérer. C'est une évolution très positive qui doit conduire à cesser de raisonner l'utilisation du coton Bt suivant l'hypothèse d'un taux de couverture de $100 \%$ (hors parcelles refuges). Dans les PED, la cohabitation du CGM avec du coton conventionnel est à rechercher pour des raisons économiques et écologiques, mais elle suppose une réorganisation de la distribution des semences qui n'est pas évidente à mettre en œuvre.

La focalisation sur la crainte de l'émergence de la résistance des ravageurs aux toxines $\mathrm{Bt}$, non encore avérée, a occulté le possible changement d'équilibre entre les ravageurs de la culture cotonnière. Aujourd'hui, une chenille vorace tend à prendre la place de la principale chenille contrôlée par le coton Bt. L'utilisation du coton Bt ne peut être la solution définitive au problème du contrôle des ravageurs. Pour être durable, son application appelle à davantage de raisonnement.

\section{BIBLIOGRAPHIE}

Asian Development Bank, 2001, «Agricultural biotechnology, poverty reduction and food security, $\mathrm{ADB}$, Manila (Philippines) », Working Paper, $225 \mathrm{p}$.

Anon, 2006a, Monsanto to develop 20 new cotton seed bybrids in India Checkbiotech.org, University of Basel.

ANON, 2006b, «Bt cotton co invites AP govt's ire", The Economic Times Online, p. 1.

BENNETT R., ISMAËL Y., et MORSE S., 2005, «Explaining contradictory evidence regarding impacts of genetically modified crops in developing countries. Varietal performance of transgenic cotton in India », Journal of Agricultural Science, 143, pp. 35-41.

BenNeTt R., ISMAËL Y. et MORSE S., 2004, "Reductions in insecticide use from adoption of Bt cotton in South Africa: impacts on economic performance and toxic load to the environment», Journal of Agricultural Science, 142, pp. 665-674.

BENNETT R., ISMAËL Y., KAMBHAMPATI U. et Morse S., 2004, "Economic impact of genetically modified cotton in India », AgBioForum 7, pp. 96-100.

Chaudhry R., 2002, «Impact of genetically engineered cotton in the world ", in Second Meeting of the Asian Cotton research and Development Network Tashkent, Uzbekistan, p. 4.

\section{Conner A. J., Glare T. R. et Nap} J.-P., 2003, « The release of genetically modified crops into the environment. Part II. Overview of ecological risk assessment », The Plant Journal, 33, pp. 1946. 
DevraJ R., 2006, «A mindless conviction. Despite failures and warnings, GM foods find a supportive government », India Together.

ELLIESEN T., 2003, « Gain in Indian cotton yield? The futile dispute over genetic engineering », $D+C$, 30, pp. 207-209.

FAO, 2004, La situation mondiale de l'alimentation et de l'agriculture 20032004. Les biotechnologies agricoles: une réponse aux besoins des plus démunis?, FAO, Rome. Collection Agriculture $\mathrm{N}^{\circ} 35$, pp. 227.

Falck-Zepeda J. B., TraXler G., and NeLSON R. G., 1999, Rent creation and distribution from the first three years of planting BT cotton, ISAAA, New York, $\mathrm{N}^{\circ} 14-1999$, pp. 18.

FitT G. P., 2003, Deployment and impact of transgenic Bt cotton in Australia, in The economic and environmental impacts of Agbiotech, New York, Kluwer, pp. 141-164

Fitt G. P., Wilson L., Mensah R. et DALY J., 2004, Advances with integrated pest management as a component of sustainable agriculture: the case of the Australian cotton industry, 4th International Crop Science Congress Brisbane, Australia, pp. 11

FoK M., LIANG W., WANG G., et WU Y., 2005, «Diffusion du coton génétiquement modifié en Chine : leçons sur les facteurs et limites d'un succès ", Économie Rurale, 285, pp. 5-32.

GALA R., 2005, « GM Cotton: corruption, hype, half-truths and lies ", in Institute of Science in Society Press Release.

Gianessi L. P., Silver C. S., SanKULA S. et CARPENTER J. E., 2002, Plant biotechnology: Current and potential impact for improving pest management in U.S. agriculture. An analysis of 40 case studies. Insect Resistant cotton, NCFAP, 20 p.

Gouse M., Kirsten J. et Beyers L., 2002, Bt Cotton in South Africa: adop- tion and the Impact on farm incomes amongst small-scale and large scale farmers. Department of Agricultural Economics, Extension and Rural development, University of Pretoria, Pretoria, Working Paper, $\mathrm{N}^{\circ}$ 2002-15, p. 13.

HOFS J.-L., FOK M., GOUSE M. et KIRSTEN J., 2006, « Diffusion du CGM en Afrique du Sud : des leçons pour l'Afrique Zone Franc", Revue Tiers Monde, $\mathrm{N}^{\circ} 188$, pp. 799-823.

Hofs J.-L., Fok M. et Vaissayre M., 2006, Impact of Bt cotton adoption on pesticide use by smallholders: A 2-year survey in Makhatini Flats (South Africa), Crop Protection, 25, pp. 984-988.

huang J., Hu R., Pray C. E., Qiao F. et Rozelle S., 2003, Biotechnology as an alternative to chemical pesticides: a case study of Bt cotton in China, Agricultural Economics, 29, 1, pp. 55-67.

ISAAA, 2005, Bt cotton: Crop losses due to insect pests of cotton and the costs of control.

Kelemu S., Mahuku G., Fregene M. et al., 2003, Harmonizing the agricultural biotechnology debate for the benefit of African farmers.

KeRBY T. A., 2001, "What changes in yield and quality are due to transgenic varieties? ", The cotton gin and oil mill press, pp. 5-7.

LANG S., 2006, Seven-Year glitch: Cornell warns that Chinese GM cotton farmers are losing money due to secondary pests.

LAws F., 2004, « Monsanto to seek arbitration on licensing dispute. Delta and Pine Land says arbitration filing is "without merit" ", Deltafarm Press, p. 2 .

LI F., 2004, «Prodenia litura (Fabricius): factors of its outbreak and how to control it », Anbui Agriculture, 7, p. 20. 
MacDonald N., 2003, Genetically Modified organisms - the last thing the developing world needs.

MAGAÑa J.E.M., GARCIA J.-G., RODRIGUEZ A.J.O. et GARCIA J.M.O., 1999, «Comparative analysis of producing transgenic cotton varieties versus nontransgenic variety in Delicias, Chihuahua, Mexico ", Beltwide Cotton Conferences, pp. 255-256

MAZOYer M., 2000, "La moitié de la paysannerie mondiale n'est pas solvable pour les grands laboratoires ", Le Monde, édition électronique du 16/10/2000.

MYers D., 1999, «GM cotton fails to impress ", Pesticides News, 44, 6.

OrPHAL J., 2005, « Comparative analysis of the economics of $\mathrm{Bt}$ and Non-Bt cotton production ", Institute of economics in Horticulture, Univ. of Hannover, Hannover, Germany, Pesticide Policy Project Publication Series. Special Issue, $71 \mathrm{p}$.

Pinstrup-Andersen P. et Schioler E., 2001, «Seeds of contention: world hunger and the global controversy over genetically modified crops ", IFPRI, Washington D.C., Food Policy Statement, 2 p.

Prakash C.S., 2004, «Global Hunger, Can Agricultural Biotechnology Help? », in Feeding a Hungry World: The Moral Imperative of Biotechnology, Rome, Italy, p. 7.

Pray C., Bengali P. et Ramaswami B., 2005, "The cost of biosafety regulations: the Indian experience ", Quaterly Journal of International Agriculture, 44, 3, pp. 267-289.

Pray C. E., huang J., Hu R., and Rozelle S., 2002, Five years of Bt cotton in China - the benefits continue, The Plant Journal 31, 4, pp. 423-430.

QAIM M., 2003, «Bt cotton in India: Field trial results and economic projec- tions ", World Development, 30, 12, p. 2115-2127.

QAim M. et JANVRY (DE) A., 2003, «Genetically modified crops, corporate pricing strategies, and Farmers' adoption: the case of Bt cotton in Argentina », Amer. J. Agr. Econ., 85, 4, p. 814828.

QAYUM A. and SAKKHARI K., 2005, " $B t$ cotton in Andhra Pradesh. A Threeyear assessment. The first ever sustained independent scientific study on Bt cotton in India ", Deccan Development society, Zabeerabad. $\mathrm{N}^{\circ}$ April 12, 2005 , pp. 55.

RAO K. C., 2003, « The ballgame of Bt cotton in India ", Foundation for Biotechnology Awareness and Education, Bangalone, India.

Russell D. et Deguine J. P., 2006, «Durabilité de la culture des cotonniers transgéniques en Chine et en Inde", Cabier d'agriculture, 2006, 15, 3, p. 5459.

Thirtle C. et Jenkins Beyers L., 2003, «Can GM-technologies help African smallholders? The impact of Bt cotton in the Makhatini Flats of KwazuluNatal», World development, 31, 4, p. $717-732$.

Traxler G., and Godoy-Avila S., 2004, Transgenic cotton in Mexico, AgBioForum 7, 1\&2, pp. 57-62.

Trigo E., Chudnovsky D., CaP E. et LOPEZ A., 2003, « Los transgénicos en la agricultura argentina. Una historia con final abierto, International Institute for Sustainable Development, Winnipeg (Canada)», Trade Knowledge Network Paper, 109 p.

Xu J., You Z., WANG W. et YANG Y., 2004, «Economic analysis of Bt cotton Planting in Jiangsu », Journal of Yangzhou University (Agricultural and Life Science Edition), 25, 3, p. 65-69. 